\title{
Performance of a Double-cantilever Dynamic Vibration Absorber (DCDVA) Oriented in Transverse and Longitudinal Directions
}

\author{
Surajudeen Adewusi \\ Department of Mechanical Engineering, Jubail University College, Royal Commission for Jubail, Saudi Arabia
}

Received July 8, 2020; Revised August 21, 2020; Accepted September 11, 2020

\begin{abstract}
Cite This Paper in the following Citation Styles
(a): [1] Surajudeen Adewusi, "Performance of a Double-cantilever Dynamic Vibration Absorber (DCDVA) Oriented in Transverse and Longitudinal Directions," Universal Journal of Mechanical Engineering, Vol. 8, No. 5, pp. 265 - 275, 2020. DOI: 10.13189/ujme.2020.080504.
\end{abstract}

(b): Surajudeen Adewusi (2020). Performance of a Double-cantilever Dynamic Vibration Absorber (DCDVA) Oriented in Transverse and Longitudinal Directions. Universal Journal of Mechanical Engineering, 8(5), 265 - 275. DOI: 10.13189/ujme.2020.080504.

Copyright $\bigcirc 2020$ by authors, all rights reserved. Authors agree that this article remains permanently open access under the terms of the Creative Commons Attribution License 4.0 International License

\begin{abstract}
This paper presents the vibration attenuation performance of a double-cantilever dynamic vibration absorber (DCDVA) attached to a simply-supported beam in two different orientations using theoretical and experimental methods. The results showed that the longitudinal configuration of the DCDVA yield better vibration attenuation by absorbing $80 \%$ more vibration than the transverse configuration. For the experimental results, the orientation of the DCDVA has very little effect on the resonant frequencies of the combined DCDVA and simply-supported beam system, however the damping of the second resonant frequency is higher for the transverse configuration. The lumped-mass model characterized the frequency response functions of the DCDVA oriented in the transverse direction only, the model could not reproduce the experimental response for the longitudinal orientation of the DCDVA.
\end{abstract}

Keywords Double-cantilever Vibration Absorber, Vibration Attenuation, Modal Parameters, Frequency Responses, Lumped-mass Model, Different Orientations

\section{Introduction}

Rotating machines such as gas and steam turbine generators, pumps, compressors, wind turbines, sometimes experience high vibration when they are in operation. For safety purposes, some rotating machines have in-built condition monitoring system (CMS), which continuously monitors vibration level and other operation parameters to prevent catastrophic failure. The CMS usually has control monitor with the ability to automatically shut down a rotating machine if the vibration level exceeds a pre-determined safe limit. Unscheduled shut down of rotating machines will result in reduction of production and loss of revenue in the industries hence vibration level is usually controlled to reduce the amplitude pending the time the machine will be shut down for maintenance or overhaul. If the machine can be operated at different speeds, reduction or increase of the machine speed may reduce the vibration amplitude. Dynamic Vibration Absorber (DVA) is one of the common vibration attenuation devices that is used to reduce vibration amplitude of machines that are running at a constant speed due to its efficiency, simplicity, reliability and low cost.

The simplest DVA was developed by Frahm in 1911 and it consists of a single degree-of-freedom mass and spring system. It is widely discussed in the majority of Mechanical Vibration textbooks [1]. The natural frequency of the DVA is designed such that it is equal to the frequency at which the machine vibration amplitude is high. The majority of subsequent research papers focused on theoretical modelling, simulation and control of different types of DVAs using lumped mass-spring-damper systems 
$[2,3,4,5,6]$. However, these lumped mass-spring-damper models are difficult to implement on machines hence in practice, a piece of mass and a thin cantilever beam, which gives flexural stiffness, are used.

Tana et. al. [7] used piezoelectric cantilever beam as autoparametric vibration absorber (AVA) for vibration suppression and energy harvesting. Both theoretical model and experiments were used to simulate and verify the performance of the system. Approximate analytical solutions using the harmonic balance method and the method of multiple scales were employed to perform synergy optimization of the system. Nonlinear saturation principle and 1:2 internal resonance were also used in the study. Wong et. al. [8] used both numerical and experimental analysis to study the effectiveness of combined translational and rotational vibration absorbers to suppress vibration of a beam subjected to a point and distributed harmonic excitations. The translational absorber consists of a spring and a cylindrical block of copper of mass $13 \mathrm{~g}$ while the rotational absorber consists of two rectangular aluminum plates of total mass $13 \mathrm{~g}$ attached to the end of a steel beam of dimensions $100 \mathrm{~mm} \mathrm{x}$ $18.3 \mathrm{~mm} \times 0.7 \mathrm{~mm}$. The other end of the steel beam was fixed to the upper top side of a horizontal flexible beam such that the rotational vibration absorber formed a cantilever beam in the vertical direction. It is not clear why the arrangement is referred to as rotational vibration absorber. For a system to have rotational degree-of-freedom, there should be angular displacement, which requires the steel beam to be rigid and attached with a pin-joint to the horizontal flexible beam.

A recent research investigated the application of dynamic vibration absorber for vibration attenuation and energy harvesting simultaneously [9]. The collocated periodic vibration absorber-harvester (PVAH) consists of two cantilever beams connected together by a discrete spring. Piezoelectric Lead Zirconate Titanate (PZT) layers were attached to a fraction of the length of the two cantilever beams for energy harvesting purposes. The performance of the PVAH was theoretically and experimentally investigated by defining absorption and harvesting indicators and their dependencies on system parameters such as load resistance. The results showed that PVAH performance is better than the conventional dynamic vibration absorber when the excitation is periodic. The study demonstrated the possibility of a DVA to have dual functions. The arrangement of the cantilever beam vibration absorber with PZT strip in [9] is similar to the arrangement of the "rotational absorber" in [8], the only difference is that no mass was attached at the end of the cantilever beam in [9].

Although lumped-mass mathematical models are very useful for simulations and to study the effect of change in model parameters on the model performance, they are grossly inadequate to represent the effect of different configuration and/or orientation of the components of the physical mechanical system. Adewusi et al. [10,11,12] used biomechanical and finite element models, instead of the lumped-mass model, of the human hand-arm to study the effect of different postures on the modal parameters and frequency responses of the human hand-arm. The studies demonstrated that the effect of hand-arm posture cannot be studied with the lumped-mass models reported in the ISO 10068 (1998) Standard [13]. Therefore, since there are different possible configurations/arrangements of the double-cantilever dynamic vibration absorber (DCDVA), it is important to investigate if the lumped-parameter model of DCDVA can adequately characterize the dynamic responses of a vibrating system with the DCDVA for different configurations. DCDVA is used in the present study, unlike a single cantilever DVA that was used in $[8$, 9], because one cantilever DVA may cause unbalance and additional vibration in the system. To the best knowledge of the author, no study has reported the effect of longitudinal and transverse arrangements of the DCDVA on the dynamic responses and modal parameters of the system.

The main aim of the present study is to investigate the effect of orientation of a double-cantilever vibration absorber on its vibration attenuation performance and modal parameters. Experiments will be performed to determine the frequency responses and modal parameters of a DCDVA and simply-supported flexible beam system when the DCDVA is oriented longitudinally and transversely to the simply-supported flexible beam. Furthermore, dynamic characterization of a lumped-mass mathematical model of the DCDVA and the simply-supported flexible beam system will be simulated for comparison with the experimental results.

\section{Methodology}

\subsection{Experimental Set-up}

GUNT TM 150 Universal Vibration System is used for the experiments. Fig. 1(a) shows the first orientation of the double-cantilever beam and absorber mass $\left(m_{2}\right)$ in the transverse direction to the simply-supported flexible beam and Fig. 1(b) presents the computer with the data acquisition system. The vibration signal from the proximity probe is connected to the GUNT TM 150.20 interface box, which in turn is connected to the PC. The speed of the harmonic exciter is controlled with the TM 150 Controller. TM 150.20 vibration software [14] was used to record and analyze the vibration signals from the proximity probe. The software permits export of the data to excel file.

Fig. 2(a) shows the longitudinal configuration of the DCDVA. The simply-supported beam, the harmonic exciter unit, support for cantilever beams and cantilever beams without the two absorbers masses will be 
represented by lumped-parameters mass $M_{1}$, stiffness $K_{1}$ and the damping coefficient $C$ for the theoretical modeling of the system. The absorber mass is made up of two pieces of rectangular steel blocks that are attached to the cantilever beam with two M6 cheese-head bolts. The parameters of the DCDVA are obtained from the absorber mass $m_{2}$ and the flexural stiffness of the cantilever beam $k_{2}$. The thickness of the cantilever beam is $1.5 \mathrm{~mm}$ hence its mass and damping are negligibly small compared to other components. The dimensions and masses of the simply-supported beam, vibration absorber and other different components of the experiment apparatus as reported in the manual [15] are summarized in Table 1. Fig. 2(a) also shows the arrangement of the vibration sensor, proximity probe, which is used to measure the vibration of the simply-supported beam. Fig. 2(b) shows the schematic diagram for the absorber masses and their distance $L$ from the cantilever support.

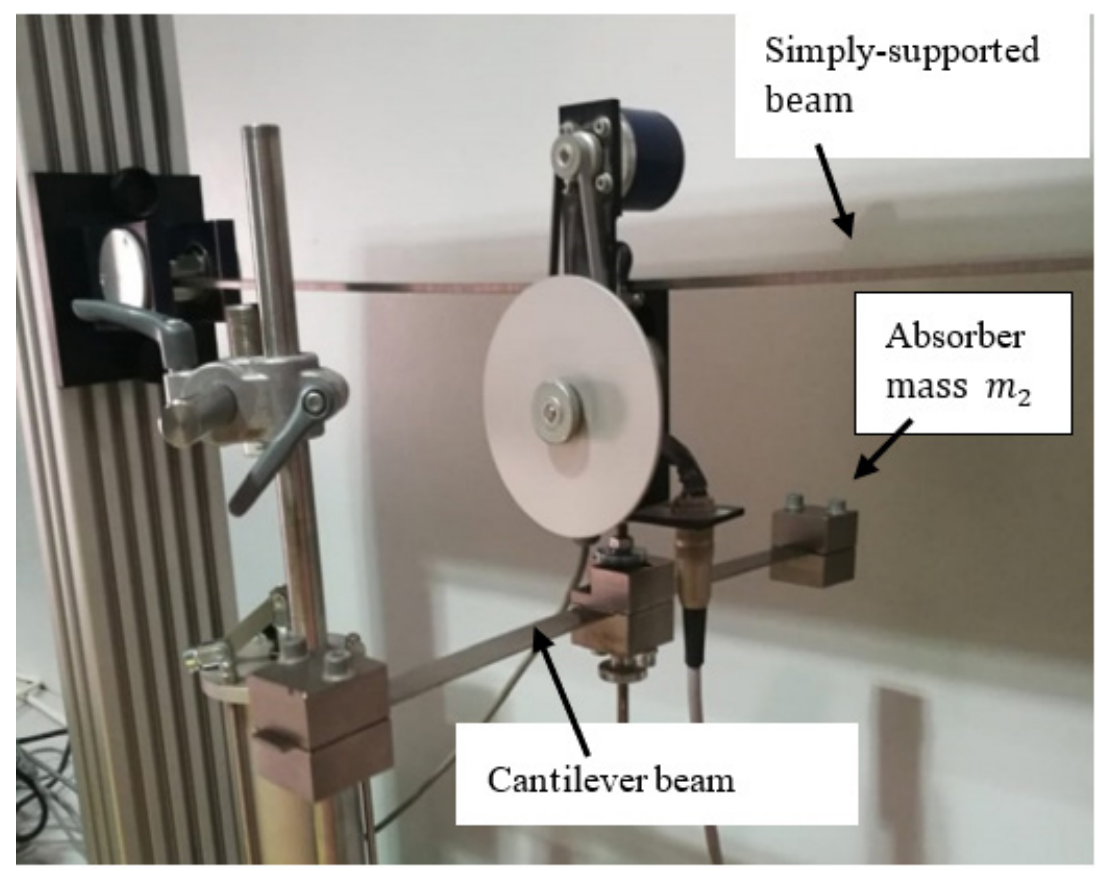

(a)

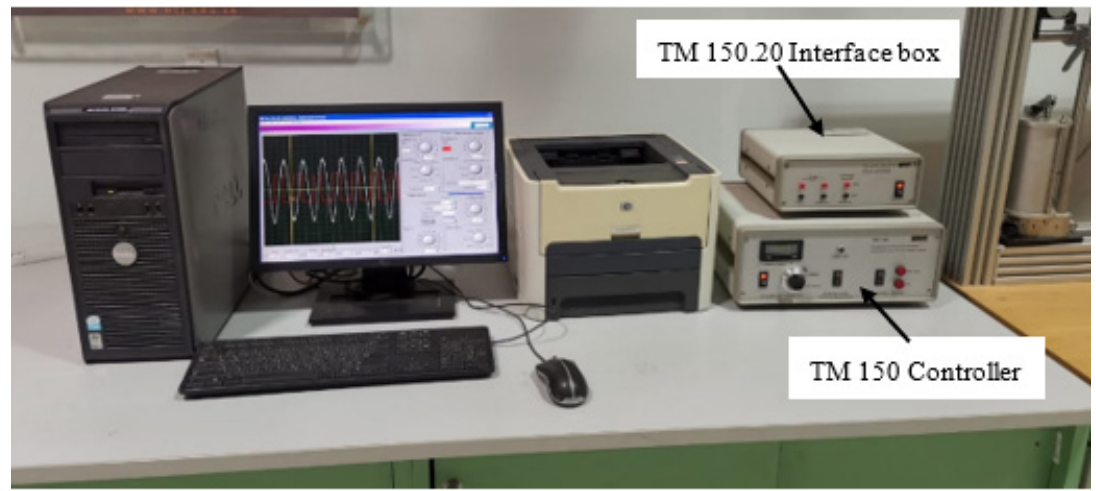

(b)

Figure 1. Experimental set-up; (a) Transverse configuration of the DCDVA, (b) Computer and data acquisition systems 


\subsection{Experimental Procedures}

For each configuration of the DCDVA, frequency response graphs were obtained for two cases viz: (1) Simply-supported beam and accessories without the absorber masses, and (2) Simply-supported beam and accessories with the absorber masses. The software automatically records and plots the response graphs on the computer monitor and can be printed. The response data are exported as excel file for further analysis. The data acquisition and analysis software plot the ratio of the proximity probe output to that of the input excitation harmonic force against the frequency in $\mathrm{Hz}$, this is the frequency response graph or the magnification factor graph. In order to ensure that the damping ratio $\zeta$ can accurately be determined from the frequency response graph, free vibration responses of the set-up in Fig. 1(a) were obtained and the damped frequency and damping ratio were obtained from the transient response graph using the concepts of periodic time $T_{d}$ and logarithmic decrement $\delta$ for comparison with the modal parameters obtained from the frequency response graphs. The results are presented in Table 2 .

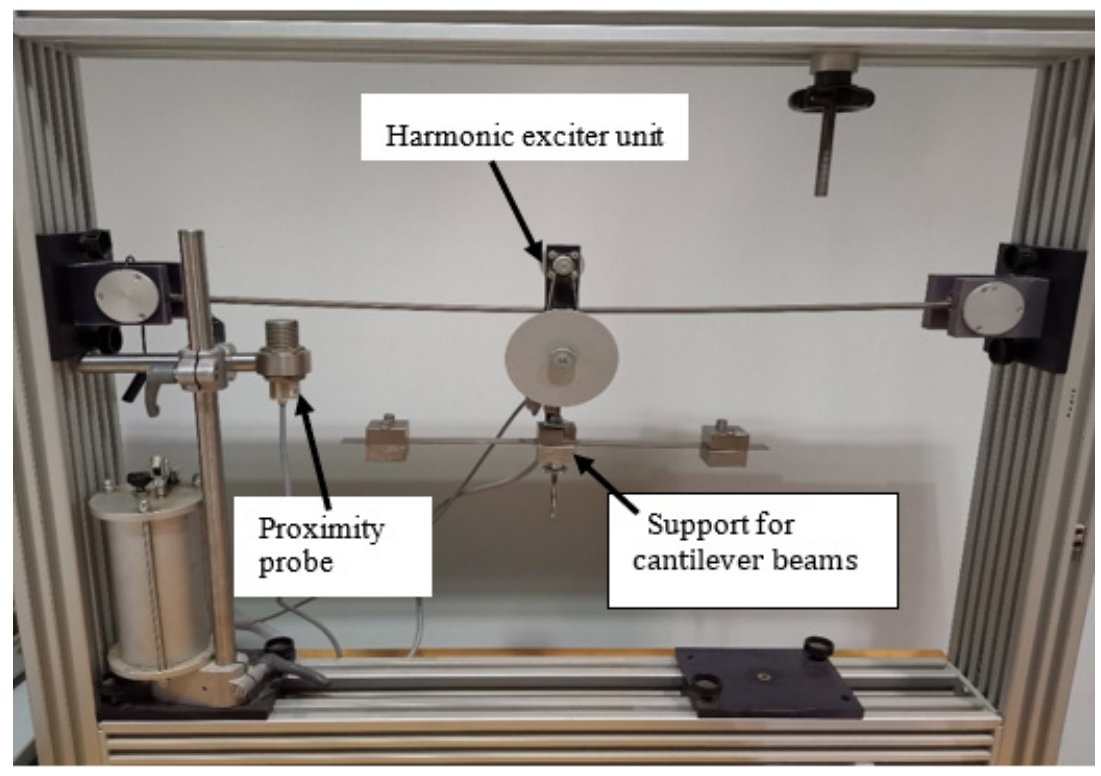

(a)

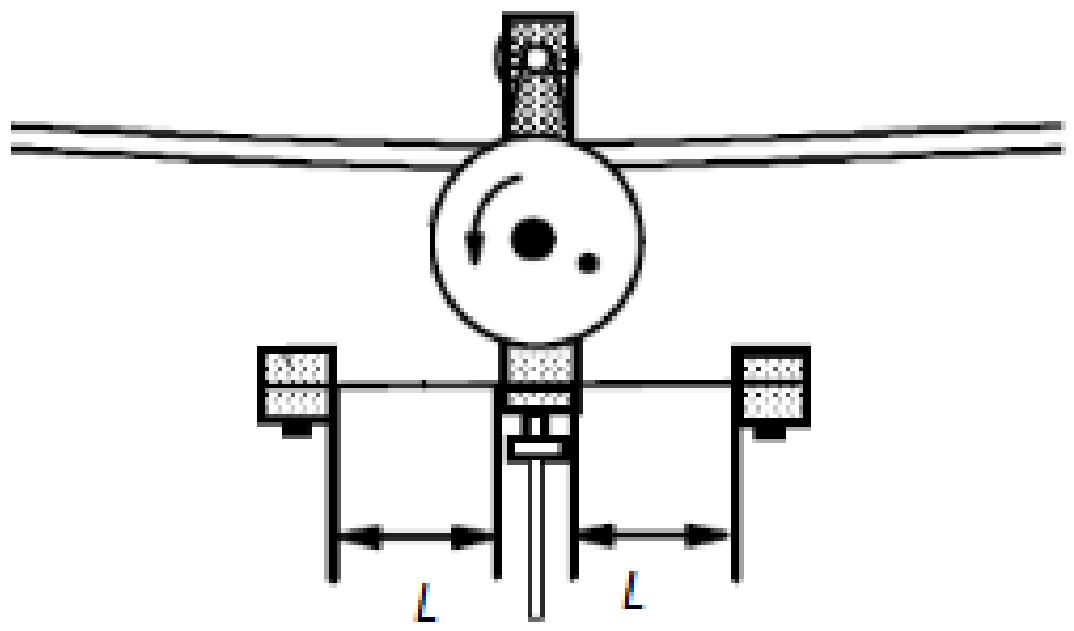

(b)

Figure 2. GUNT Universal Vibration Apparatus; (a) Longitudinal configuration of DCDVA; (b) Schematic diagram showing the distance $L$ of the absorber masses 
Table 1. Parameters of different components of the experiment apparatus

\begin{tabular}{ll}
\hline Description & Value with units \\
\hline Flexible simply-supported beam & $25 \times 4 \times 700 \mathrm{~mm}$ \\
Width x Height/Thickness x Length: & $620 \mathrm{~g}$ \\
Mass $m_{b}$ : & \\
Harmonic exciter unit & $0-50 \mathrm{~Hz}$ \\
Frequency range of the TM 150 controller $f:$ & $100 \mathrm{~g}$ \\
Unbalance mass $m_{u}:$ & $10 \mathrm{~mm}$ \\
Eccentricity $e:$ & $1000 \mathrm{~mm}-\mathrm{g}$ \\
Unbalance $m_{u-e}:$ & $772 \mathrm{~g}$ \\
Total mass $m_{e}:$ & \\
Dynamic Vibration Absorber (DVA) & $368 \mathrm{~g}$ \\
Adjustable absorber mass $m_{2}:$ & $20 \mathrm{x} 1.5 \mathrm{~mm}$ \\
Cantilever beam: Width x Height/Thickness: & $1100 \mathrm{~g}$ \\
Total mass of absorber components $(2$ cantilever beams \\
and their support and two absorber masses $\left.\left(2 m_{2}\right)\right) M_{a}:$
\end{tabular}

The free vibration response experiment for the transverse configuration of the DCDVA without the absorber masses were repeated three time and the modal parameters are summarized in Table 2. Then the forced vibration experiment was performed for the same configuration by measuring the frequency response at different frequencies to obtain the frequency response graph. The vibration amplitudes of the simply-supported flexible beam were measured at a point about $20 \mathrm{~cm}$ from the pivot end using the proximity probe.

To perform the experiments with the absorber masses attached, the distance $L$ at which the absorber masses are to be attached was first calculated by using the following equation [1]:

$$
\omega=\sqrt{\frac{3 E I}{m_{2} L^{3}}}
$$

where $\omega$ is the resonance frequency in $\mathrm{rad} / \mathrm{s}$ obtained from the frequency response graph of the system without the absorber masses, $E$ is the Young's Modulus of the material of the cantilever beam in $\mathrm{N} / \mathrm{m}^{2}, I$ is the area moment of inertia of the cross-sectional area of the cantilever beam in $\mathrm{m}^{4}$, and $L$ is the distance between the absorber masses and the support for the cantilever beam in meters. By changing $L$, the stiffness of the DCDVA and hence its natural frequency will change. The absorber masses are then fixed at the calculated length $L$ and the vibration responses are obtained again. The resonant frequencies correspond to the frequencies at which the response amplitude is maximum. The frequency response graphs are further analyzed using the half-power concept to obtain the damping ratio using the following equation [1]:

$$
Q \cong \frac{1}{2 \zeta} \cong \frac{\omega_{d}}{\omega_{2}-\omega_{1}}
$$

where $Q$ is the quality or the magnification factor, $\zeta$ is the damping ratio, $\omega_{d}$ is the damped natural frequency in $\mathrm{rad} / \mathrm{s}, \omega_{2}$ and $\omega_{1}$ are the upper and lower frequency bandwidths corresponding to the half-power points, respectively. The damped frequencies and damping ratios obtained using (2) are summarized in Table 2 presented in the Results and Discussion Section.

\subsection{Mathematical Modeling of the DCDVA and Simply-supported Flexible Beam}

Fig. 3 shows the schematic diagram of the mathematical model for the simply-supported flexible beam and the DCDVA. The entire components of the simply-supported beam are represented with lumped parameters mass $\left(M_{1}\right)$, stiffness $\left(K_{1}\right)$ and the damping coefficient $(C)$. The DCDVA is represented with absorber mass $m_{2}$ and stiffness of the one cantilever beam represented as $k_{2}$. The force due to the rotation of the harmonic exciter is represented as $F(t)$.

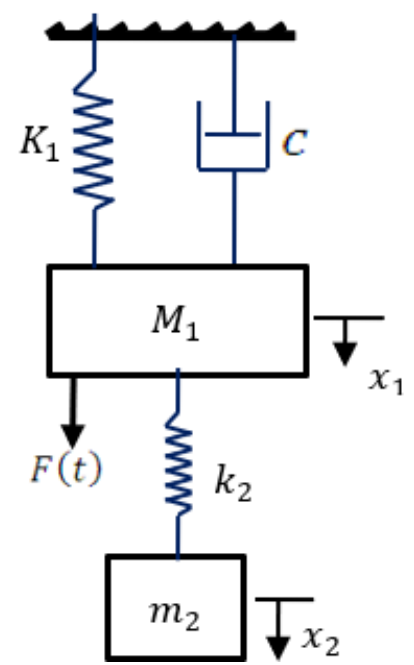

Figure 3. Schematic diagram of the model for the flexible simply-supported beam and DCDVA

The parameters of the model are obtained from values in Table 1 and the established concepts of equivalent mass 
and equivalent spring constant for simply-supported and cantilever beams in Structural Mechanics textbooks. Equations (3) through (6) are used to calculate the required model parameters. The damped frequency $\omega_{d}$ and damping ratio $\zeta$ are obtained from experimental results (damped frequency $f_{d}=7.44 \mathrm{~Hz}$ and $\zeta=0.031$ ).

$$
\begin{gathered}
M_{1}=\frac{m_{b}}{2}+m_{e}+\left(M_{a}-2 m_{2}\right) \\
K_{1}=\frac{48 E I}{l_{b}^{3}} \\
C=2 \zeta \omega_{n} M_{1} \\
k_{2}=\omega_{d}^{2} m_{2}
\end{gathered}
$$

The equations of motion for the model are:

$$
\begin{gathered}
M_{1} \ddot{x}_{1}+C \dot{x}_{1}+\left(K_{1}+k_{2}\right) x_{1}-k_{2} x_{2}=F_{o} \cos \omega t \\
m_{2} \ddot{x_{2}}+k_{2} x_{2}-k_{2} x_{1}=0
\end{gathered}
$$

By assuming harmonic responses $x_{1}=X_{1} \cos \omega t$ and $x_{2}=X_{2} \cos \omega t$, the frequency response functions of the simply-supported flexible beam $\frac{X_{1}}{F_{o}}$ and that for the absorber mass $\frac{X_{2}}{F_{o}}$ can be obtained as follows:

$$
\begin{gathered}
\frac{X_{1}}{F_{o}}=\frac{k_{2}-m_{2} \omega^{2}}{\left(k_{2}-m_{2} \omega^{2}\right)\left(\left(K_{1}+k_{2}\right)-M_{1} \omega^{2}+i C \omega\right)-k_{2}^{2}} \\
\frac{X_{2}}{F_{O}}=\frac{k_{2}}{\left(k_{2}-m_{2} \omega^{2}\right)\left(\left(K_{1}+k_{2}\right)-M_{1} \omega^{2}+i C \omega\right)-k_{2}^{2}}
\end{gathered}
$$

The damped frequencies, natural frequencies and damping ratios are obtained from the roots of the denominator of (9), which is the characteristics equation. It should be noted that the DCDVA consists of two absorber masses and two cantilever beams but $k_{2}$ and $m_{2}$ represent the properties of one beam and one mass, respectively. The question arises whether $k_{2}$ and $m_{2}$ should be multiplied by 2 in (9) and (10) or only one cantilever beam and one mass should be considered during the simulation since the DVAs are identical with symmetrical arrangement. In order to clarify the question, both cases are simulated by using $k_{2}, m_{2}$ and $2 k_{2}, 2 m_{2}$ to see the closest to the experimental responses. MATLAB program is written to simulate (9) and (10) to obtain the frequency responses and determine the modal parameters for comparison with the experimental values. Although the experimental measurement was performed on the simply-supported flexible beam represented as $x_{1}$ in Fig. 3 , the simulated responses of the mass of the DCDVA $x_{2}$ are also presented.

\section{Results and Discussion}

\subsection{Modal Parameters from the Experiments}

Figure 4 presents one of the three free vibration responses (transient responses) in time domain for the transverse configuration of the DCDVA without the absorber masses. Time Base scale of $50 \mathrm{~ms} / \mathrm{Div}$ was used to convert the horizontal distance between two peaks to seconds. From the graph, $y_{1}$ and $y_{2}$ were measured and used to calculate logarithmic decrement hence the damping ratio and natural frequency were calculated and presented in Table 2 under the heading "Time domain graphs".

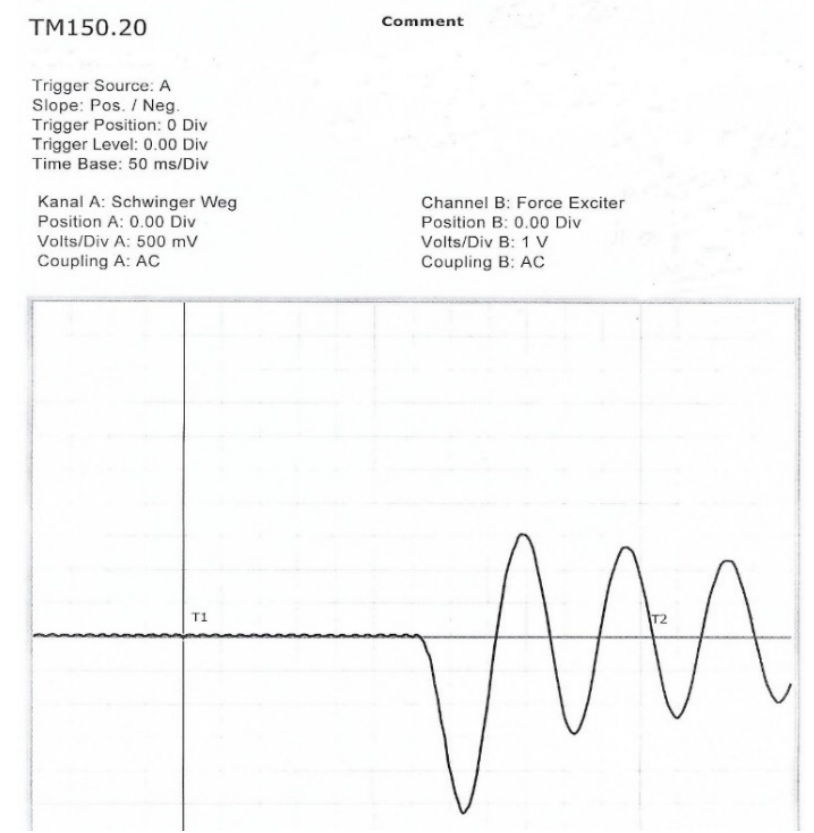

Figure 4. Free vibration response graph for transverse configuration of the DCDVA without the absorber mass

Figure 5 shows the frequency response graphs for three different experiments for the transverse configuration of the DCDVA without the absorber masses to test the repeatability of the experimental measurements. The graphs show that the vibration measurements are repeatable and consistent since there is very small deviation among the three graphs. The half-power concept was used to determine the resonant frequency and damping ratio and the values are summarized in Table 2 under the heading "Frequency response graphs". The average frequency was calculated to be $7.4 \mathrm{~Hz}, E$ of the cantilever and flexible beams is $200 \mathrm{GPa}$ and $L$ is calculated as 0.162 $\mathrm{m}$. The frequency response graphs for the transverse configuration of the DCDVA when the absorber masses were attached at $L=16.2 \mathrm{~cm}$ are presented in Fig. 6 . Unlike Fig. 5, noticeable deviations are observed among the three measurements around the second resonant frequency. This might be due to the transverse configuration of the DCDVA. A kind of random vibration similar to beat phenomenon was observed during the experiment for the transverse configuration when the absorber masses were attached. The longitudinal configuration did not exhibit any unsteady vibration.

It is observed in Fig. 6 that the resonant frequency of the simply-supported flexible beam and accessories 
slightly increased from $7.4 \mathrm{~Hz}$ to about $9.2 \mathrm{~Hz}$ where the response amplitude is minimum. The concept of DVA is to theoretical make the vibration amplitude of the simply-supported flexible beam and accessories equal to zero or very small at the resonant frequency of the system before the DVA is attached. In practice, we consider the frequency at which the amplitude is minimum as the resonant frequency of the simply-supported flexible beam and its accessories after the attachment of the absorber masses. Therefore, it is expected that the minimum amplitude in Fig. 6 will occur at the resonant frequency shown in Fig. 5, which is $7.4 \mathrm{~Hz}$. The observed difference may be due to experimental error such as the error in attaching the absorber masses at the calculated distance $L$.
$L$ has effect on the stiffness of the DCDVA and hence on the resonant frequency of the system.

Table 2 shows that the standard deviation for $f_{2}$ is the highest approximately 0.7 compared with 0.13 obtained for $f_{1}$ for the transverse configuration. The standard deviations for all the damping ratio are small and are in the range 0.004 and 0.012 . For the longitudinal configuration, one experimental measurement each was obtained for the cases without and with absorber masses, and the modal parameters obtained from the response graphs are also presented in Table 2 below the values for the transverse configuration. The vibration of the DCDVA is very stable for the longitudinal configuration, no beat phenomenon was observed.

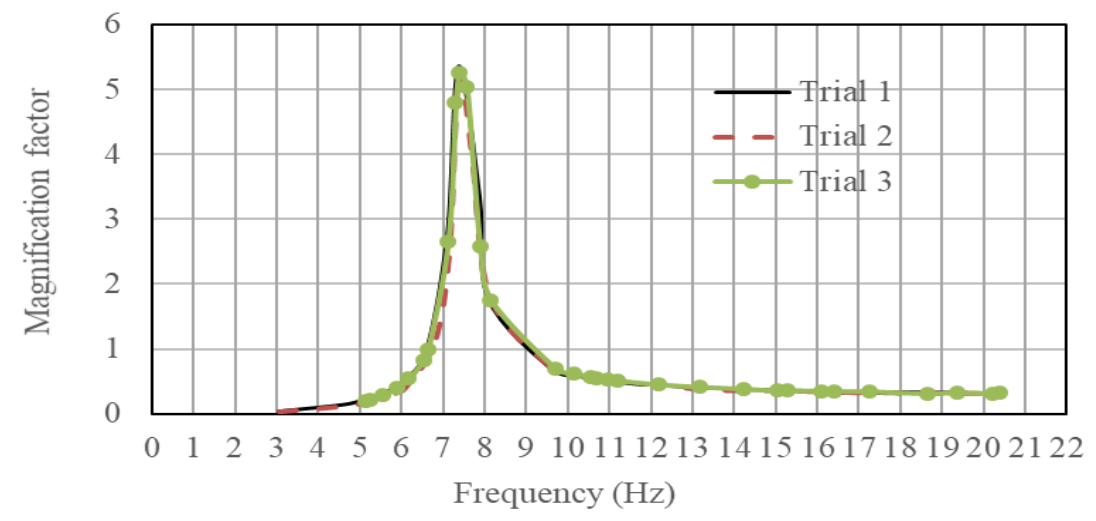

Figure 5. Repeatability of frequency response graph for transverse configuration of the DCDVA without the absorber mass

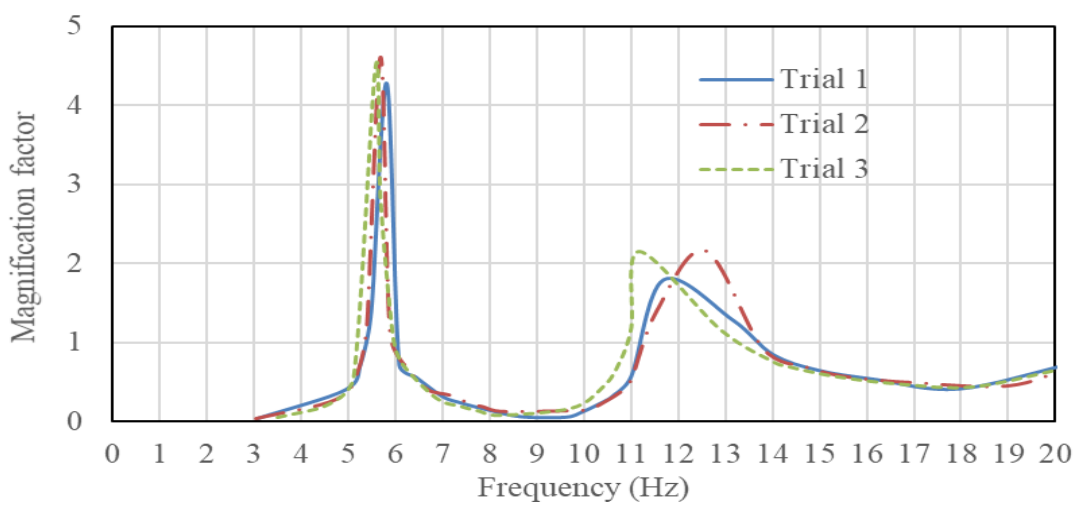

Figure 6. Repeatability of frequency response graph for transverse configuration of the DCDVA with the absorber masses

Table 2. Modal parameters from frequency response graphs for the transverse and longitudinal configurations of the DCDVA

\begin{tabular}{|c|c|c|c|c|c|c|c|c|}
\hline Description & \multicolumn{2}{|c|}{ Time domain graphs } & \multicolumn{6}{|c|}{ Frequency response graphs } \\
\hline \multicolumn{9}{|c|}{ Transverse configuration of the DCDVA } \\
\hline & \multicolumn{2}{|c|}{ Without $\boldsymbol{m}_{2}$} & \multicolumn{2}{|c|}{ Without $\boldsymbol{m}_{2}$} & \multicolumn{4}{|c|}{ With $m_{2}$} \\
\hline & $f(\mathrm{~Hz})$ & $\zeta$ & $f(\mathrm{~Hz})$ & $\zeta$ & $f_{l}(\mathrm{~Hz})$ & $\zeta_{1}$ & $f_{2}(\mathrm{~Hz})$ & $\zeta_{2}$ \\
\hline Trial 1 & 7.031 & 0.026 & 7.384 & 0.033 & 5.820 & 0.024 & 11.800 & 0.076 \\
\hline Trial 2 & 7.311 & 0.031 & 7.381 & 0.033 & 5.680 & 0.036 & 12.560 & 0.060 \\
\hline Trial 3 & 7.321 & 0.029 & 7.424 & 0.040 & 5.570 & 0.022 & 11.170 & 0.052 \\
\hline Average & 7.221 & 0.029 & 7.396 & 0.035 & 5.690 & 0.027 & 11.843 & 0.063 \\
\hline Standard Dev. & 0.165 & 0.003 & 0.024 & 0.004 & 0.125 & 0.008 & 0.696 & 0.012 \\
\hline \multicolumn{9}{|c|}{ Longitudinal configuration of the DCDVA } \\
\hline Trial 1 & \multicolumn{2}{|c|}{ NA } & 7.484 & 0.027 & 5.560 & 0.036 & 11.775 & 0.038 \\
\hline$\%$ difference & & & -1.18 & 29.63 & 2.34 & -25 & 6.80 & 65.78 \\
\hline
\end{tabular}


The percentage difference in the modal parameters between the two configurations relative to the longitudinal configuration was calculated and presented in Table 2 . The damping ratios obtained for the longitudinal configuration are approximately the same at the two resonant frequencies, while relatively high damping ratio of 0.063 for $f_{2}$ was obtained for the transverse configuration compared to 0.038 for $f_{2}$ of the longitudinal configuration. The high damping at $f_{2}$ also reduced the amplitude of the response at $f_{2}$ for the transverse configuration. The source of the additional damping for the transverse configuration needs further investigation.

Figure 7 presents the comparison of the frequency response graphs for the two configurations without and with the absorber masses in the linear scale (Fig. 7(a)) and logarithmic scale on the y-axis (Fig. 7(b)) to show the minimum value of the magnification factor and the frequency at which it occurred. Table 2 and Fig. 7 show that the orientation of the DCDVA has negligible effect on the natural frequencies but a substantial effect on the damping of the $2^{\text {nd }}$ resonant frequency of the flexible beam and DCDVA system. Fig. 7(b) shows that the minimum vibration amplitude for the system with the DCDVA occurred around $9.2 \mathrm{~Hz}$, which is higher than 7.4 $\mathrm{Hz}$, the resonant frequency of the simply-supported beam without the absorber mass for both the transverse and longitudinal configurations. The theory of DVA stipulates that minimum amplitude should be at $7.4 \mathrm{~Hz}$. The reason for this observation will be investigated with the lumped-mass model of the system. Fig. 7(b) clearly show that the longitudinal configuration of the DCDVA has higher attenuation performance than the transverse arrangement. The minimum magnification factor for the transverse and longitudinal configurations are 0.1 and 0.02 , respectively. This means that the longitudinal configuration provides $80 \%$ more vibration attenuation than the transverse configuration.

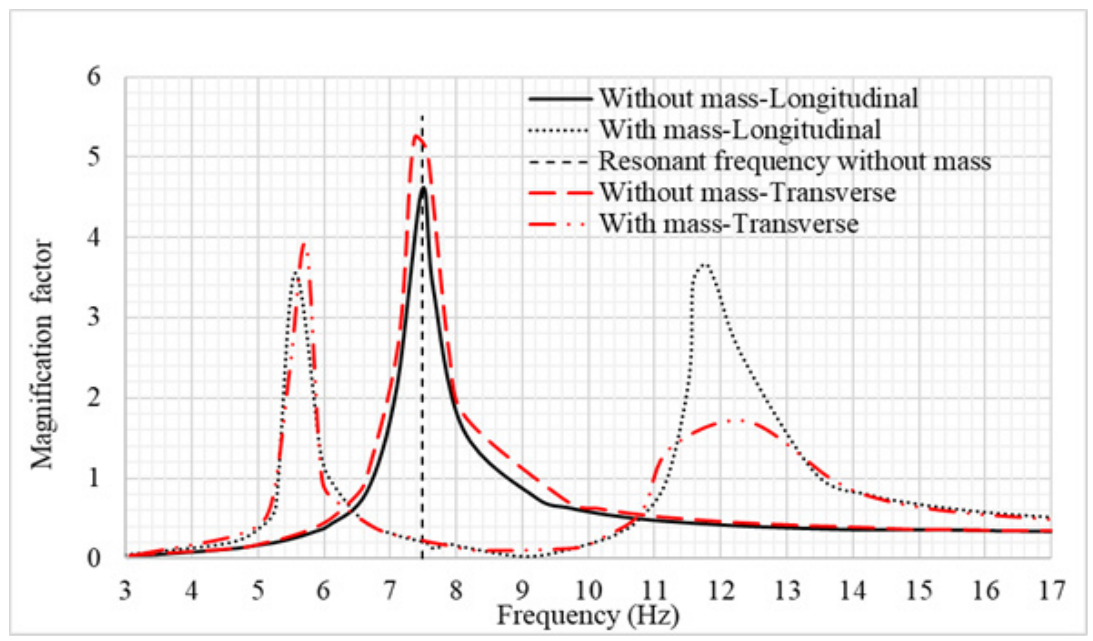

(a)

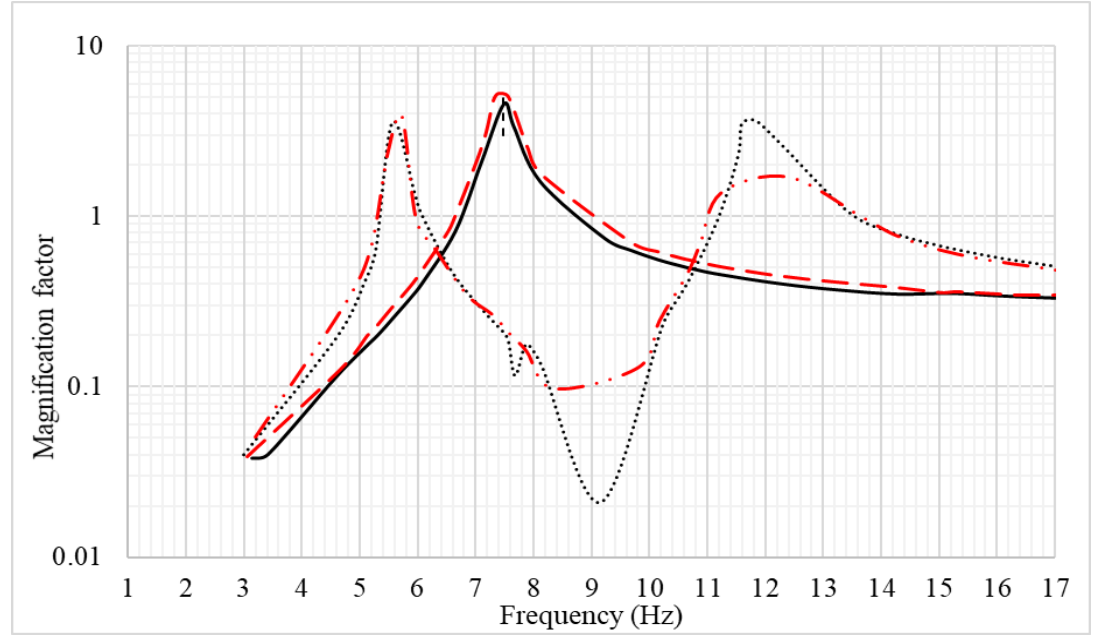

(b)

Figure 7. Comparison of frequency response graphs for transverse and longitudinal configurations of the DCDVA; (a) linear scale, (b) logarithmic $\mathrm{y}$-axis and linear $\mathrm{x}$-axis 
The frequency response for the traverse configuration in Fig. 7 is similar to that reported in $[8,9]$ in that the amplitude of the frequency responses at $f_{2}$ is lower than that at $f_{1}$. Also, the frequency response for the longitudinal configuration is similar to that reported in [15] with $f_{l}$ and $f_{2}$ having almost the same amplitude. The similarity between the frequency response graphs on page 907 of [8] and on page 341 of [9], and the transverse configuration of the DCDVA in this study suggests that the "rotational absorber" in [8] is actually a cantilever beam absorber arranged transversely to the flexible beam.

\subsection{Modal Parameters and Frequency Response Graphs of the Mathematical Model}

Figure 8 presents the frequency response graphs for the lumped-parameter model of the simply-supported flexible beam and DCDVA for two sets of the DVA parameters $\left(m_{2}, k_{2}\right.$ and $\left.2 m_{2}, 2 k_{2}\right)$. Fig. 8(a) has linear scale and Fig. 8(b) has logarithmic scale for the y-axis. The natural frequencies, damped frequencies and damping ratios were also obtained and summarized in Table 3 for comparison with the experimental values obtained for the transverse configuration of the DCDVA since Fig. 8 resembles the experimental frequency response graph for the transverse configuration. However, the damping ratio for $f_{2}$ is less in Fig. 8 compared with the value obtained from the experimental response for the transverse configuration. The damping for the model response at $f_{2}$ is almost the same as the damping at $f_{1}$. This suggests that the increase in damping for the experimental responses at $f_{2}$ may be due to the dynamics of the cantilever beam after the attachment of absorber mass. This needs further investigation, which is beyond the scope of the current study.

Unlike Fig. 7 with minimum amplitude at $9.2 \mathrm{~Hz}$, the minimum amplitude of the frequency responses for the model occurred at exactly the resonant frequency $(7.4 \mathrm{~Hz})$ of the simply-supported beam and accessories without the absorber mass. Also, Table 3 and Fig. 8 show that the parameters $\left(m_{2}, k_{2}\right)$ for one cantilever DVA should be multiplied by 2 for the modal parameters to be comparable with the experimental values obtained for the transverse configuration. This result is expected since double-cantilever DVA is used.

Table 3. Comparisons of modal parameters from experiment and model simulation

\begin{tabular}{|c|c|c|c|c|}
\hline Parameter & $\begin{array}{c}\text { Model with } \\
m_{2} \text { and } k_{2}\end{array}$ & $\begin{array}{c}\text { Model with } \\
2 m_{2} \text { and } 2 k_{2}\end{array}$ & $\begin{array}{c}\text { Transverse Configuration Experiment } \\
\text { with absorber mass }\end{array}$ & $\begin{array}{c}\text { O Difference between } \\
\text { model }\left(2 m_{2} \text { and } 2 k_{2}\right) \\
\text { and experiment values }\end{array}$ \\
\hline$M_{1}(\mathrm{~kg})$ & 1.446 & 1.446 & - & - \\
\hline$K_{1}(\mathrm{~N} / \mathrm{m})$ & 3731.778 & 3731.778 & - & - \\
\hline$C(\mathrm{Ns} / \mathrm{m})$ & 4.171 & 4.171 & - & - \\
\hline$m_{2}(\mathrm{~kg})$ & 0.368 & 0.736 & - & - \\
\hline$k_{2}(\mathrm{~N} / \mathrm{m})$ & 797.385 & 1594.770 & 11.819 & -8.22 \\
\hline$f_{n 2}(\mathrm{~Hz})$ & 9.865 & 10.847 & 5.689 & -2.94 \\
\hline$f_{n 1}(\mathrm{~Hz})$ & 6.072 & 5.522 & 11.843 & -8.42 \\
\hline$f_{d 2}(\mathrm{~Hz})$ & 9.863 & 10.846 & 5.690 & -2.95 \\
\hline$f_{d 1}(\mathrm{~Hz})$ & 6.072 & 5.522 & 0.027 & -55.56 \\
\hline$\zeta_{1}$ & 0.011 & 0.012 & 0.063 & -76.19 \\
\hline$\zeta_{2}$ & 0.016 & 0.015 & & - \\
\hline
\end{tabular}




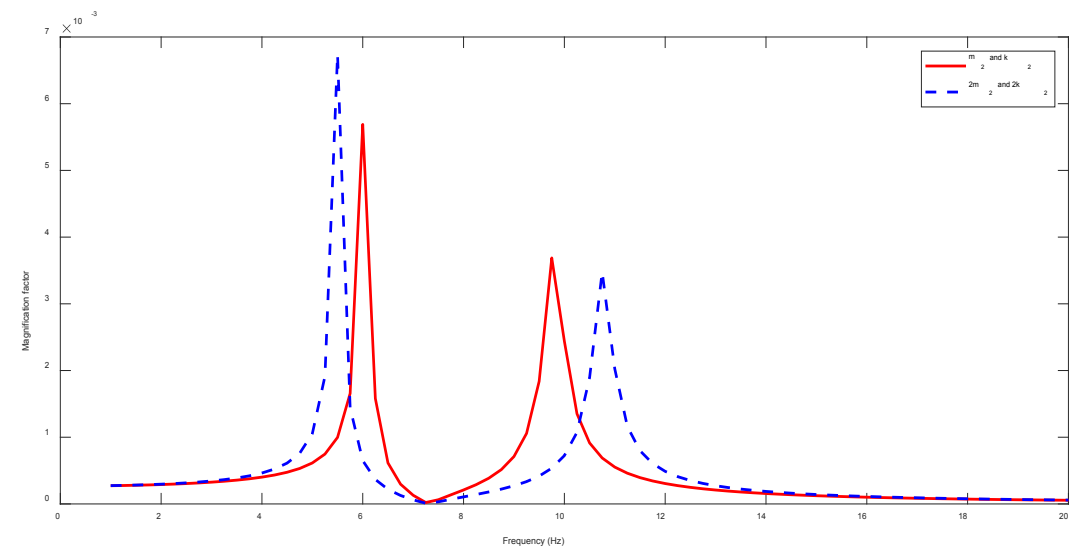

(a)

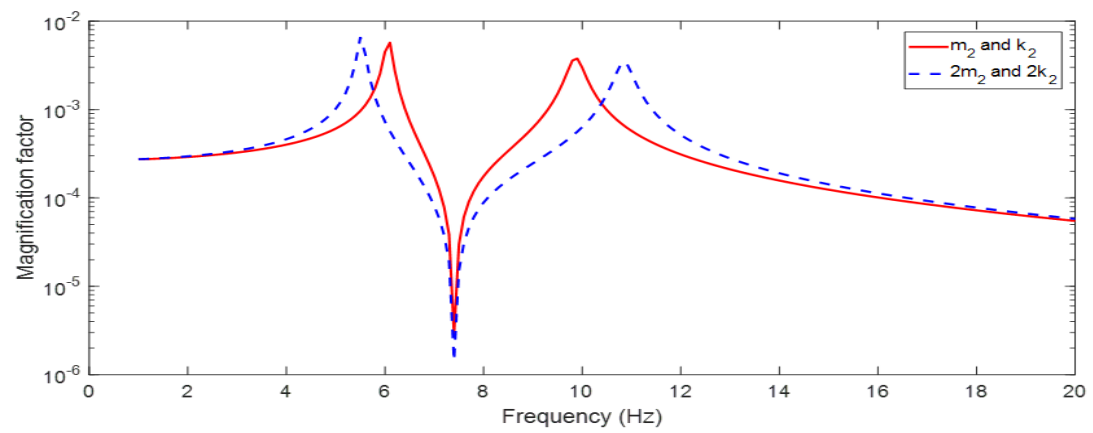

(b)

Figure 8. Comparison of frequency response graphs of the mathematical model of the beam-DCDVA system for two sets of DCDVA parameters. (a) linear scale, (b) logarithmic y-axis and linear x-axis

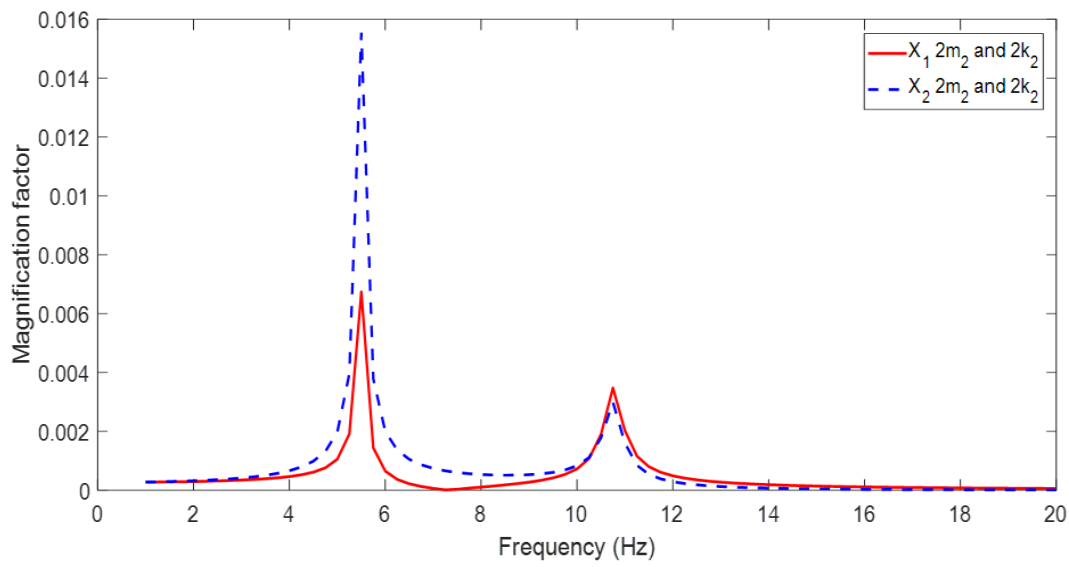

Figure 9. Comparison of frequency response graphs of the simply-supported flexible beam $\left(X_{1}\right)$ and DCDVA $\left(X_{2}\right)$ using the mathematical model of the beam-DCDVA system

\section{Conclusions}

The vibration attenuation performance of a double-cantilever dynamic vibration absorber (DCDVA) arranged in transverse and longitudinal orientations to a simply-supported flexible beam is investigated. Frequency response functions and modal parameters of the DCDVA and simply-supported beam system were obtained using both the experimental and theoretical techniques. A lumped-parameter (mass-stiffness-damper) model was used for the theoretical analysis. The longitudinal configuration of the DCDVA attained $80 \%$ more vibration attenuation than the transverse configuration. The DCDVA configuration has very little effect on the resonant 
frequencies of the combined DCDVA and simply-supported beam system. However, for the transverse configuration, the amplitude of the frequency response at the second resonant frequency is lower than the corresponding value at the first resonant frequency. The amplitude of frequency response and the damping ratios are almost the same at the two resonant frequencies for the longitudinal configuration.

The frequency responses and modal parameters of the lumped-parameter mathematical model for the system are comparable with the experimental values obtained for the transverse configuration of the DCDVA. The results showed that a lumped-parameter model is not suitable to characterize frequency responses of the DCDVA oriented in the longitudinal direction to the simply-supported beam.

\section{REFERENCES}

[1] R. Rao. Mechanical Vibrations, $5^{\text {th }}$ Edition, Prentice Hall, New York, pp. 823 - 843, 2011.

[2] T. Asami. Optimal Design of Double-Mass Dynamic Vibration Absorbers Arranged in Series or Parallel, ASME Journal of Vibration and Acoustics, Vol. 139(1), 16 pages, 2017.

[3] O. Nishihara, T. Asami. Closed-Form Solutions to the Exact Optimizations of Vibration Absorbers (Minimizations of the Maximum Amplitude Magnification Factors). ASME Journal of Vibration and Acoustics, Vol. 125(3), pp. $398-$ 405, 2002.

[4] Y. Shen, X. Wang, S. Yang, and H. Xing. Parameter Optimization for a Kind of dynamic Vibration Absorber with Negative Stiffness, Mathematical Problems in Engineering, $16 \quad$ pages, 2016. (http://dx.doi.org/10.1155/2016/9624325)

[5] C. Yang, D. Li, L. Cheng. Dynamic vibration absorbers for vibration control within a frequency band, Journal of Sound and Vibration, Vol. 330, pp. 1582 - 1598, 2011.

[6] D. A. Rade and V. Steffen Jr.. Optimization if dynamic absorbers over a frequency band, Mechanical Systems and Signal Processing, Vol. 14, pp. 679 - 690, 2000.

[7] T. Tana, Z. Yan, Z. Y. Yajian, W. Zhanga. Optimal dual-functional design for a piezoelectric autoparametric vibration absorber, Mechanical Systems and Signal Processing, Vol. 123, pp. 513-532, 2019.

[8] W. O. Wong, S. L Tang, Y. L Cheung, L. Cheng. Design of a dynamic vibration absorber for vibration isolation of beams under point or distributed loading, Journal of Sound and Vibration, Vol. 301(3-5), pp. 898-908, 2007.

[9] S.C. Huang, L. Nguyen, J. Liang, Y. Huang. Design and analysis of a collocated periodic vibration absorber-harvester, International Journal of Mechanical Sciences, Vol. 148, pp. 337-351, 2018

[10] S. Adewusi, S. Rakheja, and P. Marcotte, Biomechanical Models of the Human Hand-arm to Simulate Distributed Biodynamic Responses for Different Postures, International Journal of Industrial Ergonomics Vol. 42, 249-260, 2012.

[11] S. Adewusi, M. Thomas, V. H. Vu and W. Li. Modal Parameters of the Human Hand-arm using Finite Element and Operational Modal Analysis, Mechanics \& Industry, Vol. 15(6), 541-549, 2014.

[12] S. Adewusi, S. Rakheja, P. Marcotte and M. Thomas. Distributed vibration power absorption of the human hand-arm system in different postures coupled with vibrating handle and power tools, International Journal of Industrial Ergonomics, Vol. 43, 363 - 374, 2013.

[13] International Standards Organization, ISO 10068, Mechanical Vibration and Shock-Free Mechanical Impedance of the Human Hand-arm System at the Driving Point, 1998.

[14] Instruction Manual - TM 15x.20 Software for Data Acquisition GUNT Geratebau GmbH, hamburg, 2003.

[15] Experiment Instructions - TM 150.20 Universal Vibration System, GUNT Geratebau GmbH, hamburg. Publication No.: 941.000.00A 15012. 